\title{
Cauda equina syndrome from lumbar disc herniation
}

\author{
Drew Alexander Bednar MD
}

Cauda equina syndrome is a complex of low-back pain, bilateral sciatica, saddle anesthesia and motor weakness in the lower extremities ${ }^{1}$

The syndrome can progress to paraplegia with rectal and urinary incontinence and represents a surgical emergency. ${ }^{1}$ Sixty percent of patients are male with a mean age of 42 years, and $82 \%$ have a history of chronic low-back pain. In two-thirds of patients, the syndrome is associated with trauma or injury. ${ }^{2}$ However, cauda equina syndrome should be considered in patients whose chronic back pain has rapidly escalated regardless of a history of trauma or injury (Figure 1). ${ }^{1}$

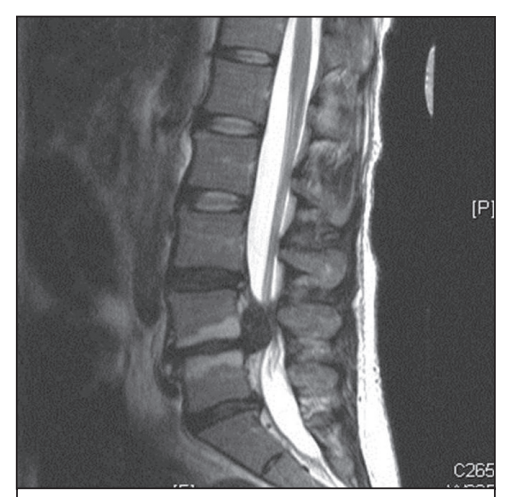

Figure 1: Mid-sagittal $T_{2}$-weighted magnetic resonance imaging (MRI) showing massive prolapse of the L4-L5 disc, causing cauda equina syndrome.
Up to $30 \%$ of patients may present without back pain

These patients may present with a sudden onset of numbness that may escalate, leg weakness or difficulty walking. ${ }^{3}$ Pain, when present, is often severe and difficult to control. Motor deficits can be profound, most commonly affecting the feet and ankles, but can also occur at the knee. Examination should include assessment for perianal numbness and digital rectal examination to detect deficits in the distal sacral nerve roots distribution (S2-S4).

\section{References}

1. Olivero WC, Wang H, Hanigan WC, et al. Cauda equina syndrome (CES) from lumbar disc herniation. J Spinal Disord Tech 2009;22:202-6.

2. Ahn UM, Ahn NU, Buchowski JM, et al. Cauda equina syndrome secondary to lumbar disc herniation: a meta-analysis of surgical outcomes. Spine 2000;25:1515-22.

3. Shapiro S. Medical realities of cauda equina syn-
Urinary or fecal retention are as common as incontinence 4

Urinary symptoms (largely S2-mediated) can include altered urethral sensation, loss of desire to void, poor stream, feeling of retention and straining to void. Perineal symptoms (S2-S4) may or may not include numbness or pain. ${ }^{5}$ Although incontinence is an obvious symptom, urinary retention with or without overflow in the bladder rendered flaccid from sacral roots paresis is not; therefore, the patient at risk should be asked about this point.

drome secondary to lumbar disc herniation. Spine 2000;25:348-51.

4. McCarthy MJ, Aylott CE, Grevitt MP. Cauda equina syndrome: factors affecting long-term functional and sphincteric outcome. Spine 2007;32:207-16.

5. Nielsen B, de Nully M, Schmidt K, et al. A urodynamic study of cauda equina syndrome due to lumbar disc herniation. Urol Int 1980;35:167-70.
Although most presentations (70\%) are acute, a more gradual onset can also occur ${ }^{3}$

Most patients (70\%) will present with severe back and leg pain rather than numbness and incontinence. ${ }^{1}$ The syndrome often evolves subacutely over days and sometimes weeks. ${ }^{3}$

Urinary incontinence from cauda equina syndrome may be reversible

Surgical treatment within 48 hours after onset brings a $70 \%$ probability that lost bladder function will be regained at two years; with delayed care, the probability is only $40 \% .^{2}$ Up to $90 \%$ of patients who receive timely treatment may regain lost bladder function at five years. ${ }^{1} \mathrm{Mag}$ netic resonance imaging should be ordered immediately on consideration of the diagnosis, since it is the standard for imaging soft tissue of the spine. Computed tomography is much less accurate and should be done only if immediately available and MRI is not, and only if it would not delay access to MRI.

\section{Competing interests: None declared.}

This article has been peer reviewed.

Affiliation: Department of Surgery, McMaster University, Hamilton, Ont.

Correspondence to: Drew Bednar, drewbednar@hotmail.com

CMAJ 2016. DOI:10.1503/cmaj.150206 